\title{
Thermoelectric transport coefficients of quark matter
}

\author{
Aman Abhisek $^{1, \mathrm{a}}$, Arpan Das ${ }^{2, \mathrm{~b}}$, Deepak Kumar ${ }^{1,3, \mathrm{c}}$, Hiranmaya Mishra ${ }^{1, \mathrm{~d}}$ (]) \\ ${ }^{1}$ Theory Division, Physical Research Laboratory, Navrangpura, Ahmedabad 380 009, India \\ ${ }^{2}$ Institute of Nuclear Physics Polish Academy of Sciences, 31-342 Kraków, Poland \\ ${ }^{3}$ Indian Institute of Technology Gandhinagar, Gandhinagar, Gujarat 382 355, India
}

Received: 6 May 2021 / Accepted: 6 January 2022 / Published online: 24 January 2022

(C) The Author(s) 2022

\begin{abstract}
A thermal gradient and/or a chemical potential gradient in a conducting medium can lead to an electric field, an effect known as thermoelectric effect or Seebeck effect. In the context of heavy-ion collisions, we estimate the thermoelectric transport coefficients for quark matter within the ambit of the Nambu-Jona Lasinio (NJL) model. We estimate the thermal conductivity, electrical conductivity, and the Seebeck coefficient of hot and dense quark matter. These coefficients are calculated using the relativistic Boltzmann transport equation within relaxation time approximation. The relaxation times for the quarks are estimated from the quark-quark and quark-antiquark scattering through meson exchange within the NJL model. As a comparison to the NJL model estimation of the Seebeck coefficient, we also estimate the Seebeck coefficient within a quasiparticle approach.
\end{abstract}

\section{Introduction}

Heavy-ion collision experiments conducted at particle accelerators allow us to study the properties of fundamental constituents of nature, such as quarks and gluons. Experiments at relativistic heavy ion collider (RHIC) and large hadron collider (LHC) indicate the formation of such a deconfined medium of quarks and gluons. The partonic medium such produced behaves like a strongly interacting liquid with a small value of shear viscosity $(\eta)$ to entropy density $(s)$ ratio $(\eta / s)$, expands, cools down and undergoes a transition to the hadronic phase and finally free streams to the detector. One of the successful descriptions of the bulk evolution of such strongly interacting matter has been through relativistic hydrodynamics. Transport coefficients are important input parameters that enter in such a dissipative hydrodynamic

\footnotetext{
a e-mail: aman@prl.res.in

b e-mail: arpan.das@ifj.edu.pl

c e-mail: deepakk@prl.res.in

d e-mail: hm@prl.res.in (corresponding author)
}

description as well as in transport simulations that have been used to describe the evolution of such matter produced in a heavy-ion collision.

Hydrodynamic studies of the heavy-ion collisions suggest that the medium produced has a very small ratio of shear viscosity to entropy density $(\eta / s)$ [1-3]. It is amongst the smallest of known materials suggesting the quark-gluon plasma (QGP) formed is the most perfect fluid. The value of this ratio estimated from experiments is also found to be close to the conjectured KSS bound on the value of $\eta / s$ [3]. Just like shear viscosity determines the response to transverse momentum gradients there are other transport coefficients such as bulk viscosity, electrical conductivity, etc. which determine the response of the system to other such perturbations. Bulk viscosity [4-9] determines the response to bulk stresses. It scales with the conformal anomaly $\left(\frac{\varepsilon-3 P}{T^{4}}\right)$ and is expected to be large near the phase transition as inferred from lattice calculations $[10,11]$. The effect of such a large bulk viscosity to entropy ratio have been investigated on the particle spectrum and flow coefficients $[12,13]$. Electrical conductivity $\left(\sigma_{e l}\right)$ [14-32] is also important as the heavy-ion collisions may be associated with large electromagnetic fields. The magnetic field produced in non-central collisions has been estimated to be of the order of $\sim m_{\pi}^{2}$ at RHIC energy scales [33-40]. Such magnetic fields are amongst the strongest magnetic fields produced in nature and can affect various properties of the strongly interacting medium. They may also lead to interesting CP-violating effects such as chiral magnetic effect etc [41]. In a conducting medium, the evolution of the magnetic field depends on the electrical conductivity. Electrical conductivity modifies the decay of the magnetic field substantially in comparison with the decay of the magnetic field in vacuum. Hence the estimation of the electrical conductivity of the strongly interacting medium is important regarding the decay of the magnetic field produced at the initial stage of heavy ion collision. These transport coefficients have been estimated in perturbative QCD and effective models [19,42- 
61]. At finite baryon densities, the other transport coefficient that is relevant is the coefficient of thermal conductivity and has been studied in $[62,63]$ both in the hadronic matter as well as partonic matter.

In the present investigation, we focus on the thermoelectric response of the strongly interacting system produced in a heavy-ion collision. It is well known from a condensed matter system that a temperature gradient can result in the generation of an electric current. This is known as the Seebeck effect. Due to temperature gradient, there is a non zero gradient of charge density leading to the generation of an electric field. A measure of the electric field produced in a conducting medium due to a temperature gradient is the Seebeck coefficient which is defined as the ratio of an electric field to the temperature gradient in the limit of vanishing electric current. Seebeck effect has been extensively studied in condensed matter systems such as superconductors, quantum dots, hightemperature cuprates, superconductor-ferromagnetic tunnel junctions, low dimensional organic metals, etc [64-72]. Such a phenomenon could also be present in the thermal medium created in heavy-ion collisions. In condensed matter systems only a temperature gradient is required for thermoelectric effect as there is only one type of dominant charge carriers in these systems. In the strongly interacting medium produced in heavy-ion collision both positive and negative charges contribute to transport phenomena. For vanishing baryon chemical potential (quark chemical potential) with equal numbers of particles and antiparticles there is no net thermoelectric effect. Thus a finite baryon chemical potential (quark chemical potential) is required for the thermoelectric effect to be observed. The strongly interacting matter at finite baryon density can be produced in low energy heavy-ion collisions at finite, e.g. at FAIR and NICA. Along with the temperature gradient, we also consider a gradient in the baryon (quark) chemical potential to estimate the Seebeck coefficient of the partonic medium. The gradient in the chemical potential has effects similar to the temperature gradient. Using Gibbs Duhem relation for a static medium one can express gradient in the baryon (quark) chemical potential to a gradient in temperature. Effect of the chemical potential gradient significantly affects the thermoelectric coefficients as has been demonstrated in Ref. [73].

Seebeck effect in the hadronic matter has been investigated previously by some of us within the framework of the Hadron resonance gas model $[73,74]$. However, the Hadron resonance gas model can only describe the hadronic medium at chemical freezeout whereas one expects deconfined partonic medium at the early stages of the heavy-ion collisions. In this investigation, we estimate the thermoelectric behavior of the partonic medium within the framework of the NJL model. Seebeck coefficient has also been estimated for the partonic matter within relaxation time approximation in Ref. $[75,76]$. However, this has been attempted with the relax- ation time estimated within perturbative QCD which may be valid for asymptotically high temperatures. Further, it ought to be mentioned that, the vacuum structure of QCD remain nontrivial near the critical temperature region with nonvanishing values for the quark-antiquark condensates associated with chiral symmetry breaking as well as Polyakov loop condensates associated with the physics of statistical confinement [77-80]. Indeed, within the ambit of the NJL model, it was shown that the temperature dependence of viscosity coefficients exhibits interesting behavior of phase transition with the shear viscosity to entropy ratio showing a minimum while the coefficient of bulk viscosity showing a maximum at the phase transition $[77,78,81]$. The crucial reason for this behavior was the estimation of relaxation time using medium dependent masses for the quarks as well as the exchanged mesons which reveal nontrivial dependence before and after the transition temperature. This motivates us to investigate the behavior of thermoelectric transport coefficients within the NJL model which takes into account the medium dependence of quark and meson masses. This model has been used to study different transport properties of quark matter at high temperatures [6,31,81,82] and high densities [83-90]. Apart from the NJL model we also use quasi-particle model [9197], which provides a reasonable thermodynamic and transport behavior of the deconfined phase.

We organize the paper in the following manner. In Sect. 2, we discuss the Boltzmann equation within relaxation time approximation to have the expressions for the different thermoelectric transport coefficients in the presence of a condensate. In Sect. 3 we discuss thermodynamics and estimation of relaxation time within the two flavor NJL model. In Sect. 4 we discuss the quasiparticle approach in the absence of any quark-antiquark condensate. In Sect. 5 we present the results of different transport coefficients. Finally, we give a possible outlook of the present investigation and conclude in Sect. 6.

\section{Boltzmann equation in relaxation time approximation and transport coefficients}

Within a quasiparticle approximation, a kinetic theory treatment for the calculation of transport coefficient can be a reasonable approximation that we shall be following similar to that in Refs. [5, 6, 45,46,98,99]. The plasma can be described by a phase space density for each species of particle. Near equilibrium, the distribution function can be expanded about a local equilibrium distribution function for the quarks as,

$f(\mathbf{x}, \mathbf{p}, t)=f^{(0)}(\mathbf{x}, \mathbf{p})+\delta f(\mathbf{x}, \mathbf{p}, t)$,

where the local equilibrium distribution function $f^{(0)}$ is given as

$f^{(0)}(\mathbf{x}, \mathbf{p})=\left[\exp \left(\beta(\mathbf{x})\left(u_{v} p^{v} \mp \mu(\mathbf{x})\right)\right)+1\right]^{-1}$ 
Here, $u^{\mu}=\gamma_{u}(1, \mathbf{u})$, is the flow four-velocity, where, $\gamma_{u}=\left(1-\mathbf{u}^{2}\right)^{1 / 2} ; \mu$ is the chemical potential associated with a conserved charge. Here $\mu$ denotes the quark chemical potential and $\beta=1 / T$ is the inverse of temperature. Further, $p^{\mu}=(E, \mathbf{p})$ is the particle four momenta, single particle energy $E=\sqrt{p^{2}+M^{2}}$ with $p=|\mathbf{p}| . M$ is the mass of the particle which in general is medium dependent. The departure from the equilibrium is described by the Boltzmann equation,

$\frac{d f_{a}(\mathbf{x}, \mathbf{p}, t)}{d t}=\frac{\partial f_{a}}{\partial t}+\frac{d x^{i}}{d t} \frac{\partial f_{a}}{\partial x^{i}}+\frac{d p^{i}}{d t} \frac{\partial f_{a}}{\partial p^{i}}=C^{a}[f]$,

where we have introduced the species index ' $a$ ' on the distribution function. The right-hand side is the collision term which we shall discuss later. The left-hand side of the Boltzmann equation involves the trajectory $\mathbf{x}(t)$ and the momentum $\mathbf{p}(t)$. This trajectory, in general, not a straight line as the particle is moving in a mean-field, which, in general, can be space time-dependent. The velocity of the particle ' $a$ ' is given by

$\frac{d x^{i}}{d t}=\frac{\partial E_{a}}{\partial p_{a}^{i}}=\frac{p_{a}^{i}}{E_{a}}=v_{a}^{i}$.

Next, the time derivative of momentum, the force, in presence of an electric field $(\mathcal{E})$, magnetic field $(\mathbf{B})$ and a mean field dependent mass can be written as

$\frac{d p^{i}}{d t}=-\frac{\partial E_{a}}{\partial x^{i}}+q_{a}\left(\mathcal{E}^{i}+\varepsilon^{i j k} v_{j} B_{k}\right)$.

The time derivatives of $\mathbf{x}$ and $\mathbf{p}$ can be substituted on the left-hand side of the Boltzmann equation Eq. (2) and the same reduces to

$$
\begin{aligned}
\frac{\partial f_{a}}{\partial t}+v^{i} \frac{\partial f_{a}}{\partial x^{i}}+ & \frac{\partial f_{a}}{\partial p^{i}}\left(-\frac{M_{a}}{E_{a}} \frac{\partial M_{a}}{\partial x^{i}}+q_{a}\left(\mathcal{E}^{i}+\varepsilon^{i j k} v_{j} B_{k}\right)\right) \\
& =C^{a}[f] .
\end{aligned}
$$

For the collision term on the right-hand side, we shall be limiting ourselves to $2 \rightarrow 2$ scatterings only. In the relaxation time approximation the collision term for species $a$, all the distribution functions are given by the equilibrium distribution function except the distribution function for particle $a$. The collision term, to first order in the deviation from the equilibrium function, will then be proportional to $\delta f_{a}$, given the fact that $C^{a}\left[f^{(0)}\right]=0$ by the principle of local detailed balance. In that case, the collision term is given by

$C[f]=-\frac{\delta f_{a}}{\tau_{a}}$,

where, $\tau_{a}$, the relaxation time for particle ' $a$ '. In general relaxation time is a function of energy. We shall discuss more about it in the subsequent subsection where we estimate it within the NJL model. As an approximation of the collision kernel in the Boltzmann equation one can also use other collision terms, e.g. Chapmann-Enskog method apart from the relaxation time approximation $[4-6,44,45,47,99]$. The relaxation time approximation for the collision integral in the Boltzmann equation may not be a controlled approximation scheme. The Chapmann-Enskog method which uses a variational approach can in principle allows one to obtain solutions with arbitrary accuracy. Nonetheless, the relaxation time approximation has been used more often to evaluate transport coefficients due to its simplicity. Although Chapmann-Enskog method can be in satisfying agreement with the Green-Kubo formalism, the qualitative behavior of various transport coefficients also remains the same in the relaxation time approximation [47]. Therefore as a first step towards the estimation of the thermoelectric transport coefficient within the framework of an effective model of QCD, we stick to the relaxation time approximation. Any calculation with a more realistic collision term will be an improvement on the present results.

Returning back to the left-hand side of Eq. (3), we keep up to the first order in gradients in space-time. The left-hand side of the Boltzmann equation Eq. (3), is explicitly small because of the gradients and we, therefore, may replace $f_{a}$ by $f_{a}^{(0)}$. While the spatial derivative of the distribution function is given by,

$$
\begin{aligned}
\frac{\partial f_{a}^{(0)}}{\partial x^{i}} & =-f_{a}^{(0)}\left(1-f_{a}^{(0)}\right) \partial_{i}\left(\beta E_{a}-\beta \mu_{a}\right) \\
& =-f_{a}^{(0)}\left(1-f_{a}^{(0)}\right)\left(-\frac{E_{a}}{T^{2}} \partial_{i} T+\beta \frac{M_{a}}{E_{a}} \frac{\partial M_{a}}{\partial x^{i}}\right. \\
& \left.-\partial_{i}\left(\beta \mu_{a}\right)\right),
\end{aligned}
$$

here $\mu_{a}=b_{a} \mu, b_{a}$ being the quark number, i.e. $b_{a}=1$ for quarks and $b_{a}=-1$ for antiquarks. The momentum derivative of the equilibrium distribution function is given by,

$\frac{\partial f_{a}^{(0)}}{\partial p^{i}}=-\frac{1}{T} f_{a}^{(0)}\left(1-f_{a}^{(0)}\right) v_{a}^{i}$

Substituting Eqs. (6) and (5) in the Boltzmann equation Eq. (3) for the static case (where the distribution function is not an explicit function of time) in the absence of magnetic field we have

$$
\begin{aligned}
- & f_{a}^{(0)}\left(1-f_{a}^{(0)}\right)\left[v_{a}^{i}\left(-\frac{1}{T^{2}} \partial_{i} T E_{a}-\partial_{i}\left(\beta \mu_{a}\right)\right)+q_{a} \beta v_{a}^{i} \mathcal{E}^{i}\right] \\
& =-\frac{\delta f_{a}}{\tau_{a}} .
\end{aligned}
$$


The spatial gradients of temperature and chemical potential can be related using momentum conservation in the system and Gibbs Duhem relation. Momentum conservation in a steady-state leads to $\partial_{i} P=0$ ( $P$, being the pressure) [100]. Using Gibbs Duhem relation, the pressure gradient can be written as, with the enthalpy $\omega=\varepsilon+P$,

$\partial_{i} P=\frac{\omega}{T} \partial_{i} T+T n_{q} \partial_{i}(\mu / T)$

which vanishes in steady-state. $n_{q}$ denotes the net quark number density and $\varepsilon$ is the energy density. The above equation relates the spatial gradient of temperature to the spatial gradients in chemical potential as,

$\partial_{i} \mu=\left(\mu-\frac{\omega}{n_{q}}\right) \frac{\partial_{i} T}{T}$.

Using Eqs. (9) and (7), $\delta f_{a}$, the deviation of the distribution function is given as,

$\delta f_{a}=\frac{\tau_{a} f_{a}^{0}\left(1-f_{a}^{0}\right)}{T}\left[q_{a} \mathbf{v}_{a} \cdot \mathcal{E}-\left(E_{a}-b_{a} \frac{\omega}{n_{q}}\right) \frac{\mathbf{v}_{a} \cdot \nabla T}{T}\right]$.

The nonequilibrium part of the distribution function gives rise to transport coefficients. The electric current is now given as,

$$
\begin{aligned}
\mathbf{J} & =\sum_{a} g_{a} \int \frac{d^{3} p_{a}}{(2 \pi)^{3}} q_{a} \mathbf{v}_{a} \delta f_{a} \\
& =\sum_{a} \frac{g_{a} q_{a}^{2}}{3 T} \int \frac{d^{3} p_{a}}{(2 \pi)^{3}} v_{a}^{2} \tau_{a} f_{a}^{0}\left(1-f_{a}^{0}\right) \mathcal{E} \\
& -\sum_{a} \frac{g_{a} q_{a}}{3 T^{2}} \int \frac{d^{3} p_{a}}{(2 \pi)^{3}} \tau_{a}\left(E_{a}-b_{a} \frac{\omega}{n_{q}}\right) f_{a}^{0}\left(1-f_{a}^{0}\right) v_{a}^{2} \nabla T .
\end{aligned}
$$

In Eq. (11) we have used $v_{a}^{i} v_{a}^{j}=\frac{1}{3} v_{a}^{2} \delta^{i j}$ as because the integrand only depends on the magnitude of momenta. Further, the sum is over all flavors including antiparticles. The degeneracy factor $g_{a}=6$ corresponding to color and spin degrees of freedom. $b_{a}$ is the quark number i.e. $b_{a}= \pm 1$ for quarks and antiquarks respectively.

Next, we write down the heat current $\mathcal{I}$ associated with the conserved quark number. For a relativistic system, thermal current arises corresponding to a conserved particle number. The thermal conduction due to quarks arises when there is energy flow relative to enthalpy [100]. Therefore the heat current is defined as [100],

$\mathcal{I}^{i}=\sum_{a} T_{a}^{0 i}-\frac{\omega}{n_{q}} \sum_{a} b_{a} J_{q a}^{i}$.
Here, $n_{q}$ is the net quark number density. The energy flux is given by $T^{0 i}$, the spatio-temporal component of energymomentum tensor $\left(T^{\mu v}\right)$ [100],

$T_{a}^{0 i}=g_{a} \int \frac{d^{3} p_{a}}{(2 \pi)^{3}} p_{a}^{i} f_{a}$

while, the quark current is given $\mathbf{J}_{q}$ is given by

$J_{q a}^{i}=g_{a} \int \frac{d^{3} p_{a}}{(2 \pi)^{3}} \frac{p_{a}^{i}}{E_{a}} f_{a} b_{a}$.

Clearly, the contribution to the energy flux and quark current vanishes arising from the equilibrium distribution function $f_{a}^{(0)}$ due to symmetry consideration and it is only the nonequilibrium part $\delta f_{a}$ that contribute to the energy flux and quark current in Eqs. (13) and (14) respectively. Substituting the expression for $\delta f_{a}$ from Eq. (10) in Eq. (12), the heat current $\mathcal{I}$ is given as,

$$
\begin{gathered}
\mathcal{I}=\sum_{a} \frac{g_{a}}{3 T} \int \frac{d^{3} p_{a}}{(2 \pi)^{3}} f_{a}^{0}\left(1-f_{a}^{0}\right) v_{a}^{2} \tau_{a}\left[q_{a}\left(E_{a}-b_{a} \frac{\omega}{n_{q}}\right) \mathcal{E}\right. \\
\left.-\left(E_{a}-b_{a} \frac{\omega}{n_{q}}\right)^{2} \frac{\nabla T}{T}\right]
\end{gathered}
$$

The Seebeck coefficient $S$ is defined by setting the electric current $\mathbf{J}=0$ in Eq. (11) so that the electric field becomes proportional to the temperature gradient i.e.

$\mathcal{E}=S \nabla T$

Therefore the Seebeck coefficient for the quark matter in the presence of a gradient in temperature and chemical potential can be expressed as,

$S=\frac{\sum_{a} \frac{g_{a} q_{a}}{3 T} \int \frac{d^{3} p_{a}}{(2 \pi)^{3}} \tau_{a} v^{2}\left(E_{a}-b_{a} \frac{\omega}{n_{q}}\right) f_{a}^{(0)}\left(1-f_{a}^{(0)}\right)}{T \sum_{a} \frac{g_{a}}{3 T} q_{a}^{2} \int \frac{d^{3} p_{a}}{(2 \pi)^{3}} v^{2} \tau_{a} f_{a}^{(0)}\left(1-f_{a}^{(0)}\right)}$

The denominator of the Seebeck coefficient in the above may be identified as $T \sigma_{e l}$, where the electrical conductivity $\sigma_{e l}$ is given by $[21,101]$,

$\sigma_{e l}=\sum_{a} \frac{g_{a}}{3 T} q_{a}^{2} \int \frac{d^{3} p_{a}}{(2 \pi)^{3}}\left(\frac{p_{a}}{E_{a}}\right)^{2} \tau_{a} f_{a}^{(0)}\left(1-f_{a}^{(0)}\right)$

which may be identified from Eq. (11). Let us note that, while the denominator of the Seebeck coefficient is positive definite, the numerator is not so as it is linearly dependent on the electric charge of the species as well as on the difference $\left(E_{a}-b_{a} \frac{\omega}{n_{q}}\right)$. This makes the Seebeck coefficient not always 
positive definite. This is also observed in different condensed matter systems [102].

In terms of the electrical conductivity and the Seebeck coefficient, the electric current Eq. (11) can be written as

$\mathbf{J}=\sigma_{e l} \mathcal{E}-\sigma_{e l} S \nabla T$

In a similar manner, the heat current as given in Eq. (15) can be written as,

$\mathcal{I}=T \sigma_{e l} S \mathcal{E}-\kappa_{0} \nabla T$

where, $\kappa_{0}$, the thermal conductivity can be written as [100]

$$
\begin{array}{r}
\kappa_{0}=\sum_{a} \frac{g_{a}}{3 T^{2}} \int \frac{d^{3} p_{a}}{(2 \pi)^{3}} \tau_{a}\left(\frac{p_{a}}{E_{a}}\right)^{2}\left(E_{a}-b_{a} \frac{\omega}{n_{q}}\right)^{2} \\
f_{a}^{(0)}\left(1-f_{a}^{(0)}\right) .
\end{array}
$$

Using Eqs. (19) and (20), we can express the heat current $(\mathcal{I})$ in terms of electric current $(\mathbf{J})$ in the following way,

$\mathcal{I}=T S \mathbf{J}-\left(\kappa_{0}-T \sigma_{e l} S^{2}\right) \nabla T$

From Eq. (22) we can identify the Peltier coefficient $(\Pi)$ and thermal conductivity $(\kappa)$ in the presence of nonvanishing Seebeck coefficient as,

$\Pi=T S, \kappa=\kappa_{0}-T \sigma_{e l} S^{2}$

Note that the relation between the Peltier coefficient $(\Pi)$ and the Seebeck coefficient as given in Eq. (23) can be considered as the consistency relation. Also, note that the thermal conductivity in the absence of any thermoelectric effect as given in Eq. (21) matches with the expression of the thermal conductivity as reported in [100]. The Seebeck coefficient $(S)$, thermal conductivity $\left(\kappa_{0}\right)$, and the electrical conductivity $\left(\sigma_{e l}\right)$ depend upon, the estimation of the relaxation time as well as the quark masses that goes into the distribution functions through the single-particle energies and are medium dependent. We estimate these quantities in the Nambu-JonaLasinio model which is described in the next section.

Before we start the discussion of the relaxation time within the framework of the NJL model we should emphasize the key features of the formalism as discussed above. The formalism of the transport coefficients as presented in Ref. [81] was developed to incorporate the effect of a nonvanishing dynamical quark condensate. In the present manuscript, we incorporated the effect of a dynamical condensate in the formalism of thermoelectric effects following Ref. [81]. On the other hand, the formalism of thermoelectric transport coefficients as presented in Refs. [73,74] do not include the effect of any dynamical condensate. Note that the formalism to calculate transport coefficients using the Boltzmann equation is fundamentally different in the presence of a dynamical condensate. In the presence of such a condensate, there exists an additional effective force term (see Eq. (3)) which is not present otherwise $[73,74]$. Therefore the formalism presented here should not be considered to be the same as the formalism presented in Refs. [73,74]. Furthermore, the final expression of the Seebeck coefficient and the thermal conductivity as obtained in the present investigation exactly resembles the results as given in Refs. [73,74,81]. This is an interesting outcome despite the fact that in the present investigation we started with a Boltzmann kinetic equation which is different from the same given in Refs. [73,74]. This is because the term proportional to $\partial M / \partial x$, which also acts as an effective force term in the Boltzmann equation (see Eq. (3)), gets exactly canceled by a similar term originating from $\partial f^{(0)} / \partial x$. We should also point out that the constituent quark mass which enters into the expressions of various transport coefficients through the single particle energy also carries nontrivial temperature and chemical potential dependence due to the presence of the gap equation. Such a temperature and chemical potential dependence of the single particle energy was not present in our earlier investigations, e.g. Ref. $[73,74]$. Therefore even if the analytical expressions presented in this investigation look similar to the expressions presented in Ref. [73,74], the theoretical formalism and the temperature and chemical potential dependence of various thermoelectric transport coefficients are different.

\section{Estimation of relaxation time in NJL model}

We model the partonic medium using the two flavor NambuJona-Lasinio (NJL) model and estimate the thermodynamic quantities, the quasi particle masses in the medium and the relaxation time. The two flavour NJL model with $u$ and $d$ quark, can be described by the following Lagrangian [103],

$\mathcal{L}=\bar{\psi}\left(i \not \partial-m_{q}\right) \psi+G\left[(\bar{\psi} \psi)^{2}+\left(\bar{\psi} i \gamma^{5} \tau \psi\right)^{2}\right]$

Here, $\psi$ is the doublet of $u$ and $d$ quarks; $m_{q}$ is the current quark mass matrix which is diagonal with elements $m_{u}$ and $m_{d}$ and we take them to be same as $m_{0}$ assuming isospin symmetry; $\tau$ are the Pauli matrices in the flavor space; $G$ is the scalar coupling. NJL model is a QCD inspired effective model which incorporates various aspects of the chiral symmetry of QCD. The NJL model Lagrangian as given in Eq. (24) is symmetric under the chiral symmetry group $S U(2)_{V} \times S U(2)_{A} \times U(1)_{V}$. The thermodynamic quantities e.g., pressure $(P)$, energy density $(\varepsilon)$ and the number density $\left(n_{q}\right)$ can be obtained once we know the thermodynamic 
potential of the NJL model. In a grand canonical ensemble, the thermodynamic potential $(\Omega)$ or equivalently the pressure $(P)$ can be expressed as,

$$
\begin{aligned}
-P= & \Omega(\beta, \mu)=\frac{\left(M-m_{0}\right)^{2}}{4 G} \\
& -\frac{2 N_{c} N_{f}}{(2 \pi)^{3} \beta} \int d \mathbf{k}\left[\log \left(1+e^{-\beta(E-\mu)}\right)\right. \\
& \left.+\log \left(1+e^{-\beta(E+\mu)}\right)\right]-\frac{2 N_{c} N_{f}}{(2 \pi)^{3}} \int d \mathbf{k} \sqrt{\mathbf{k}^{2}+M^{2}} .
\end{aligned}
$$

In the above, $N_{c}=3$ is the number of colors and $N_{f}=2$ is the number of flavors, $E=\sqrt{\mathbf{k}^{2}+M^{2}}$ is the single particle energy with 'constituent' quark mass $M$ which satisfies the self consistent gap equation

$M=m_{0}+\frac{2 N_{c} N_{f}}{(2 \pi)^{3}} \int d \mathbf{k} \frac{M}{\sqrt{k^{2}+M^{2}}}\left(1-f^{(0)}-\bar{f}^{(0)}\right)$.

In the above equations $f^{(0)}=\left(1+\exp \left(\beta \omega_{-}\right)\right)^{-1}$ and $\bar{f}^{(0)}=$ $\left(1+\exp \left(\beta \omega_{+}\right)\right)^{-1}$ are the equilibrium distribution functions for quarks and antiquarks respectively and we have written $\omega_{ \pm}(k)=E(\mathbf{k}) \pm \mu$ with $k \equiv|\mathbf{k}|$. The energy density $\varepsilon$ is given by,

$\varepsilon=-\frac{2 N_{c} N_{f}}{(2 \pi)^{3}} \int d \mathbf{k} E(k)\left(1-f^{(0)}-\bar{f}^{(0)}\right)+\frac{\left(M-m_{0}\right)^{2}}{4 G}$,

so that enthalpy $\omega=\varepsilon+P$ is also defined once the solution to the mass gap equation Eq. (26) is known. In these calculations, we have taken a three momentum cutoff $\Lambda$ for the for calculations of integrals not involving the Fermi distribution functions. The net number density of quarks $n_{q}$ is given as

$n_{q}=\frac{2 N_{c} N_{f}}{(2 \pi)^{3}} \int d \mathbf{k}\left(f^{(0)}-\bar{f}^{(0)}\right)$.

This completes the discussion on the all the bulk thermodynamic quantities defined for NJL model which enters in the definitions for Seebeck coefficient, electrical conductivity and thermal conductivity.

Next we discuss the estimation of relaxation time and as mentioned earlier we consider two particle scattering processes only. For a process $a+b \rightarrow c+d$, the relaxation time for the particle $a$ i.e. $\tau_{a}\left(E_{a}\right)$ is given by [81],

$\tau_{a}^{-1}\left(E_{a}\right) \equiv \tilde{\omega}\left(E_{a}\right)=\frac{1}{2 E_{a}} \sum_{b} \int d \pi_{b} W_{a b} f_{b}^{(0)}\left(E_{b}\right)$,

where, the summation is over all species other than the particle " $a$ ". Further, in Eq. (29), we have introduced the notation $d \boldsymbol{\pi}_{i}=\frac{d^{3} p_{i}}{(2 \pi)^{3} 2 E_{i}}$ and $W_{a b}$ is the dimensionless transition rate for the processes with $a, b$ as the initial states. $W_{a b}$ which is Lorentz invariant and a function of the Mandelstam variable $(s)$ can be given by,

$$
\begin{aligned}
W_{a b}(s)= & \frac{1}{1+\delta_{a b}} \int d \boldsymbol{\pi}_{c} d \boldsymbol{\pi}_{d}(2 \pi)^{4} \delta\left(p_{a}+p_{b}-p_{c}-p_{d}\right) \\
& \times|\mathcal{M}|_{a b \rightarrow c d}^{2}\left(1-f_{c}^{(0)}\left(p_{c}\right)\right)\left(1-f_{d}^{(0)}\left(p_{d}\right)\right) .
\end{aligned}
$$

In the expression of $W_{a b}$ the Pauli blocking factors have been considered. The quantity $W_{a b}$ can be related to the cross sections of various scattering processes. In the present case within the NJL model, the quark-quark, quark-antiquark and antiquark-antiquark scattering cross sections are calculated to order $1 / N_{c}$ which occur through the $\pi$ and $\sigma$ meson exchanges in " $s$ " and " $t$ " channels. The meson propagators that enters into the scattering amplitude is calculated within the random phase approximation and includes their masses and the widths. The mass of the meson is estimated from the pole of the meson propagator at vanishing three momentum i.e.,

$1-2 G \operatorname{Re} \Pi_{\tilde{m}}\left(M_{\tilde{m}}, 0\right)=0$.

where $\tilde{m}$ denotes $\sigma, \pi$ for scalar and pseudoscalar channel mesons, respectively. Polarization function in the corresponding mesonic channel is expressed as $\Pi_{\tilde{m}}$. The explicit expressions for $\operatorname{Re} \Pi_{\tilde{m}}$ and the imaginary $\operatorname{part} \operatorname{Im} \Pi_{\tilde{m}}$ is given in Ref. [81] and we do not repeat here.

While, the relaxation time is energy dependent, one can also define an energy independent mean relaxation time by taking a thermal average as,

$\bar{\omega}_{a} \equiv \bar{\tau}_{a}^{-1}=\frac{1}{n_{a}} \int \frac{d^{3} p_{a}}{(2 \pi)^{3}} f_{a}^{(0)}\left(E_{a}\right) \tilde{\omega}_{a}\left(E_{a}\right) \equiv \sum_{b} n_{b} \bar{W}_{a b}$,

to get an estimate of the average relaxation time. In the above equation, the sum is over all the particles other than ' $a$ ';

$n_{a}=\int \frac{d^{3} p_{a}}{(2 \pi)^{3}} f_{a}^{(0)}\left(E_{a}\right)$,

is the number density of the species " $a$ " apart from the degeneracy factor. Here, $\bar{W}_{a b}$ is the thermal-averaged transition rate given as

$\bar{W}_{a b}=\frac{1}{n_{a} n_{b}} \int d \pi_{a} d \pi_{b} f\left(E_{a}\right) f\left(E_{b}\right) W_{a b}$.

For the case of two flavors, there are 12 different processes but the corresponding matrix elements can be related using ispin symmetry, charge conjugation and crossing symmetries with only two independent matrix elements. We have chosen 
them, as in Refs. [81,99], to be the processes $u \bar{u} \rightarrow u \bar{u}$ and $u \bar{d} \rightarrow u \bar{d}$. The explicit expressions for the matrix elements are given in Refs. [81,99]. In the meson propagators we have kept both the mass and the width of the meson resonances which are medium dependent. It is important to mention that while the matrix elements of different scattering processes are related, the thermal-averaged rates are not. This is because the thermal averaged rates involve also the thermal distribution functions for the initial states along with the Pauli blocking factors for the final states.

\section{Quasiparticle picture of the partonic medium}

In the quasiparticle description, all the quarks (anti quarks) have both the thermal mass arising due to the interaction with the constituents of the medium and the bare mass $m_{0}$. Therefore, in the quasiparticle picture the total effective mass of the quark flavor $i$ can be expressed as [91-97,104],

$m_{i}^{2}=\left(m_{0}+m_{+}(T, \mu)\right)^{2}+m_{+}(T, \mu)^{2}$,

with

$2 m_{+}^{2}(T, \mu)=\frac{g^{2}(T, \mu) T^{2}}{3}\left(1+\frac{\mu^{2}}{\pi^{2} T^{2}}\right)$.

which is related to the asymptotic form of the gauge independent hard thermal (dense) loop self energies. The temperature and the chemical potential dependent strong coupling constant up to two loop order is $[105,106]$,

$$
\begin{aligned}
\alpha_{S}(T, \mu)= & \frac{g^{2}(T, \mu)}{4 \pi}=\frac{6 \pi}{\left(33-2 N_{f}\right) \ln \left(\frac{T}{\Lambda_{T}} \sqrt{1+\frac{\mu^{2}}{\pi^{2} T^{2}}}\right)} \\
& \times\left(1-\frac{3\left(153-19 N_{f}\right)}{\left(33-2 N_{f}\right)^{2}} \frac{\ln \left(2 \ln \frac{T}{\Lambda_{T}} \sqrt{1+\frac{\mu^{2}}{\pi^{2} T^{2}}}\right)}{\ln \left(\frac{T}{\Lambda_{T}} \sqrt{1+\frac{\mu^{2}}{\pi^{2} T^{2}}}\right)}\right),
\end{aligned}
$$

where $\Lambda_{T}$ is the QCD scale parameter which we consider as $\Lambda_{T}=0.35 T_{c}$ with $T_{c}=200 \mathrm{MeV}$ [106]. The effective mass of the gluon, which depends on the temperature and the chemical potential is given as $[96,107]$,

$m_{g}^{2}(T)=\frac{N_{c}}{6} g^{2}(T, \mu) T^{2}\left[1+\frac{1}{6}\left(N_{f}+\frac{3}{\pi^{2}} \sum_{f} \frac{\mu_{f}^{2}}{T^{2}}\right)\right]$,

where $N_{c}$ and $N_{f}$ represents the number of color and flavors respectively. The relaxation time for the quarks and antiquarks for the massless case is given by [108],

$$
\tau_{q(\bar{q})}=\frac{1}{5.1 T \alpha_{S}^{2} \log \left(\frac{1}{\alpha_{S}}\right)\left(1+0.12\left(2 N_{f}+1\right)\right)} .
$$

Note that for simplicity we have used the relaxation time which is applicable for the massless case only. However, following Ref. [109] it can be argued that the effect of the massive quark is small in the estimation of the scattering cross sections and relaxation time. Therefore, we use the expressions of the relaxation time as given in Eq. (38) even for the massive quarks. To compare our results as obtained in the NJL model we consider two light flavors with bare mass $m_{0}=0.008 \mathrm{GeV}$ [96]. The relaxation time for the gluons is given by $[104,108,110]$

$$
\tau_{g}=\frac{1}{22.5 \alpha_{s}^{2} \ln \left(\frac{1}{\alpha_{s}}\right)\left(1+0.06 n_{f}\right)} .
$$

\section{Results}

The two flavor NJL model as given in Eq. (24) has three parameters, the four fermions coupling $G$, the three momenta cut off $(\Lambda)$ to regularize the momentum integral in vacuum and the current quark mass $m_{0}$. These values are adjusted to fit the physical values of the pion mass $\left(m_{\pi}=135 \mathrm{MeV}\right)$, the pion decay constant $\left(f_{\pi}=94 \mathrm{MeV}\right)$ and the value of the quark condensate in vacuum, $\langle\bar{u} u\rangle=\langle\bar{d} d\rangle=(-241 \mathrm{MeV})^{3}$. Various combinations of $G, \Lambda, m_{0}$ can be used to fix the pion mass, pion decay constant, and the quark vacuum condensate. Qualitatively all these different parameterizations are equivalent. Without going into such parameter dependence we work with a single set of parameters. We have considered here the value of the parameters as $m_{0}=5.6 \mathrm{MeV}, \Lambda=587.9 \mathrm{MeV}$ and $G \Lambda^{2}=2.44$ [103]. This leads to the constituent quark mass for $u$ and $d$ type quarks, $M=397 \mathrm{MeV}$ in vacuum $(T=0, \mu=0)$.

To analyze the variation of different transport coefficients with temperature and quark chemical potential, we have first plotted in upper panel of Fig. 1, the constituent quark masses $(M)$ as a function of temperature $(T)$ for different values of the quark chemical potential $(\mu)$. The constituent quark mass $(M)$ results as a solution to the gap equation, Eq. (26). Constituent quark masses for $u$ and $d$ quarks are the same and they are related to the quark-antiquark condensate $\langle\bar{\psi} \psi\rangle$. In the lower panel of Fig. 1, we have plotted $d M / d T$ with temperature for different values of the chemical potential. For the range of temperature and chemical potential considered here the chiral transition is a smooth crossover. The chiral crossover temperature may be defined by the position of the peak in the variation of $d M / d T$ with temperature. For $\mu=$ 0,100 and $200 \mathrm{MeV}$, the corresponding chiral crossover temperatures turns out to be $\sim 188 \mathrm{MeV}, 180 \mathrm{MeV}$ and $153 \mathrm{MeV}$ 

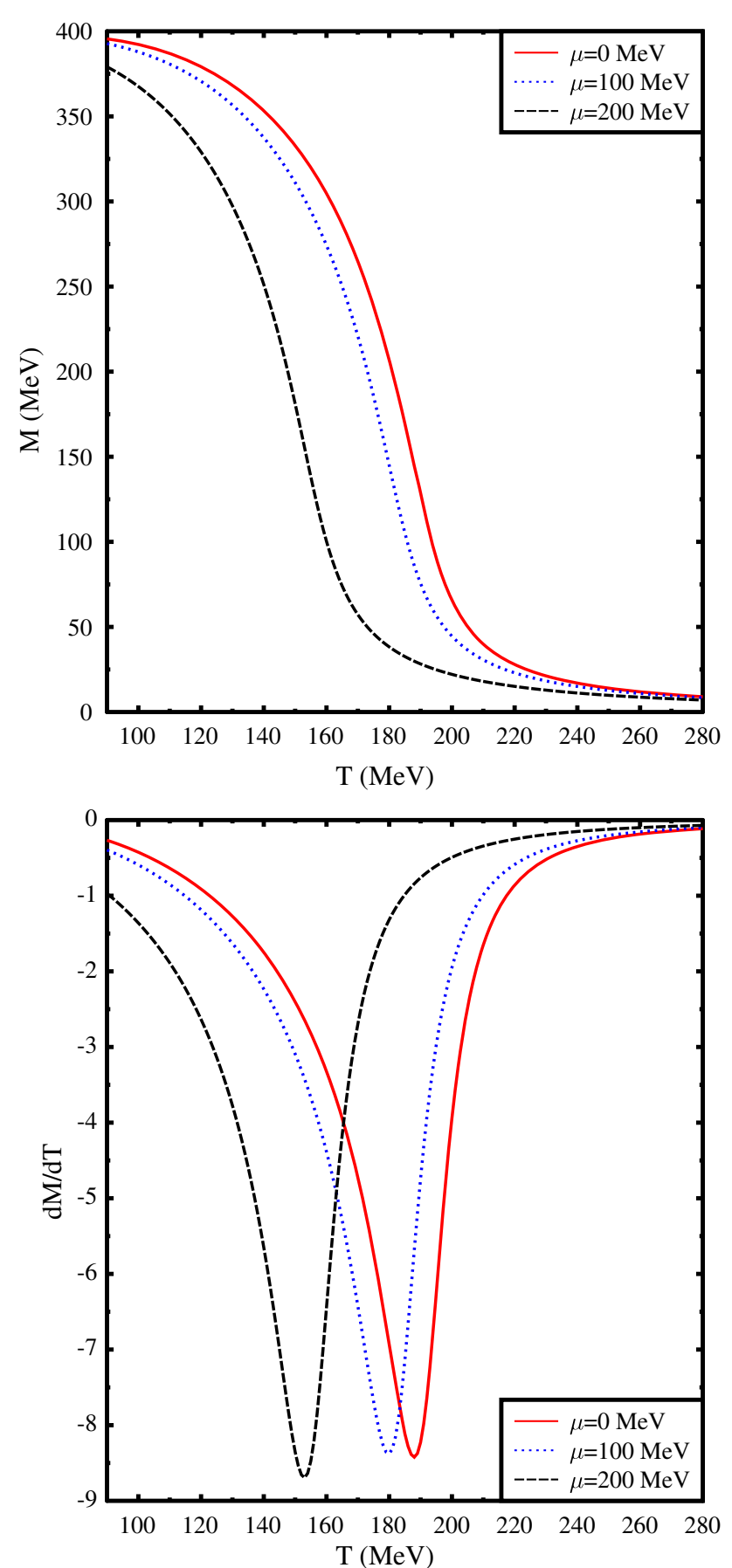

Fig. 1 Upper panel: temperature dependence of the masses of constituent quarks $(M)$ for different chemical potentials. Lower panel: variation of $d M / d T$ with temperature for different chemical potentials. The nonmonotonic variation of $d M / d T$ with a peak structure indicate the pseudo critical temperature for the chiral transition. Note that for the NJL model parameter set and the range of temperature and chemical potential considered here the chiral transition is a smooth crossover

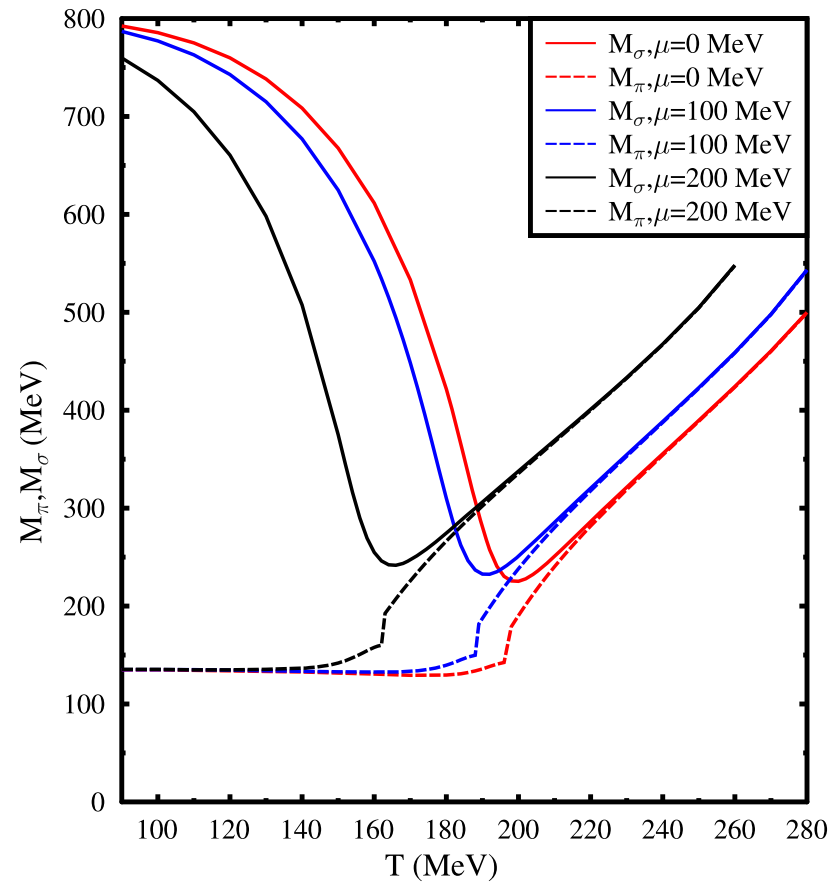

Fig. 2 Variation of $\sigma$ and $\pi$ meson masses with temperature for different values of the chemical potentials. The solid lines correspond to $M_{\sigma}$ while the dashed lines correspond to pion masses, $M_{\pi}$

respectively. It is expected that with an increase in chemical potential the crossover temperature decreases. Note that we have considered here the values of the chemical potential which are lower than the chemical potential corresponding to the speculated critical endpoint of the quark-hadron phase transition in the QCD phase diagram.

In Fig. 2 we have plotted the meson masses $M_{\pi}$ and $M_{\sigma}$ as a function of temperature for different values of chemical potential as solutions of Eq. (31). Note that pions are pseudoGoldstone modes, therefore in the chiral symmetry broken phase pion mass varies weakly. But $M_{\sigma}$ decreases rapidly near the crossover temperature. At higher temperatures, $M_{\pi}$ and $M_{\sigma}$, being chiral partners, become approximately degenerate and increase with temperature. Further one can define a characteristic temperature, the "Mott temperature" $\left(T_{M}\right)$ where the pion mass becomes twice that of quark mass i.e. at Mott temperature $M_{\pi}\left(T_{M}\right)=2 M\left(T_{M}\right)$. The Mott temperatures for $\mu=0,100$ and $200 \mathrm{MeV}$ turns out to be $\sim 198$ $\mathrm{MeV}, 192 \mathrm{MeV}$ and $166 \mathrm{MeV}$ respectively. As we shall see later it is the Mott temperature that becomes relevant while estimating the relaxation times of the quarks using thermal scattering rates of the quarks through meson exchange.

In Fig. 3, we show the variation of average relaxation time as defined in Eq. (32), for quarks (solid lines) and antiquarks(dashed lines) with temperature for different chemical potentials. Let us note that the relaxation time of given particle ' $a$ ', as shown in Eq. (32), depends both on the scattering rates $\bar{W}_{a b}$ as well as on the number density $n_{b}$ of the 


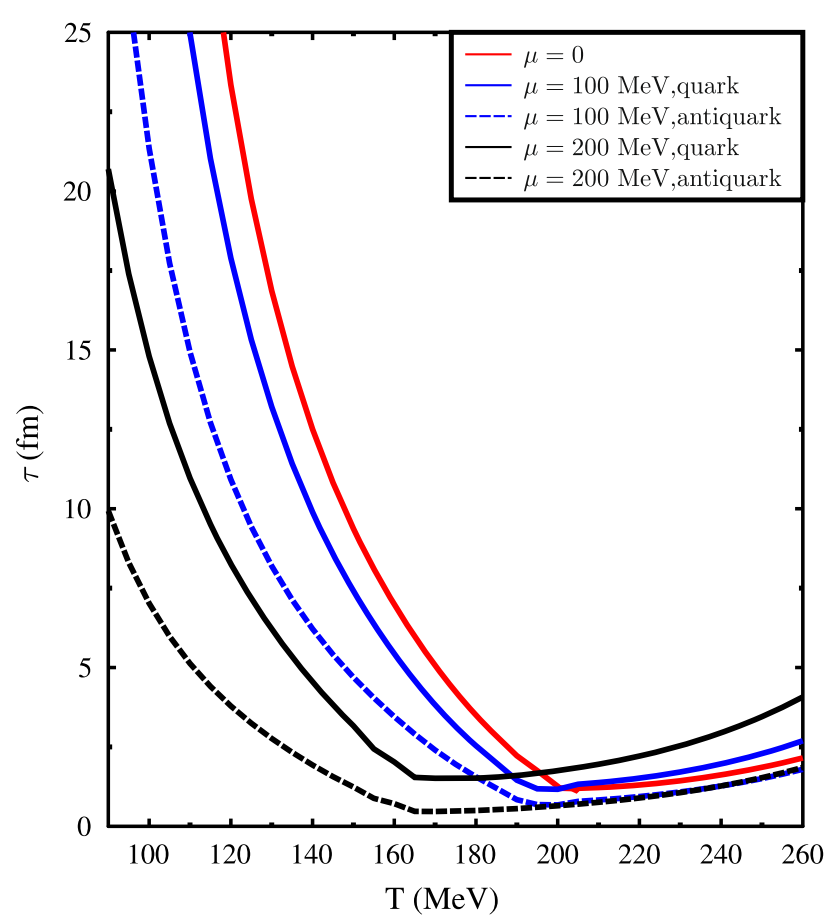

Fig. 3 Variation of thermal averaged relaxation times for quarks and antiquarks with temperature for different chemical potentials. Solid lines correspond to the relaxation time for quarks while the dotted lines correspond to relaxation time for antiquarks. For $\mu=0$ the thermal averaged relaxation times for the quarks and antiquarks are same. Difference between the relaxation times of quarks and antiquarks appears only at finite chemical potential

particles other than ' $a$ ' in the initial state i.e. number density of scatterers. It turns out that, for the scattering processes considered here, the process $u \bar{d} \rightarrow u \bar{d}$ [81], through charged pion exchange in the s-channel gives the largest contribution as compared to other channels. As mentioned earlier,by crossing symmetry arguments, this also means that the $u d \rightarrow u d$ scattering rate also contribute dominantly to the thermally averaged scattering rate. Let us note that, below the the Mott temperature $T_{M}$, the averaged scattering rate decreases mostly due to thermal distribution with large constituent quark masses apart from the suppression from the meson propagators in the scattering amplitudes arising from sigma mesons. As one approaches $T_{M}$ from lower temperature, the scattering rates become larger as the constituent quark mass decreases as well as the s-channel propagator develop a pole in the meson propagator for temperatures beyond $T_{M}$. However, at large temperature there will be a suppression due to the large meson masses which increase with temperature. This results in a maximum scattering rate at $T_{M}$ or a minimum in the average relaxation time as generically seen in Fig. 3.

At finite quark chemical potentials, beyond the Mott temperature, the quark-antiquark scattering still contributes dominantly to the scattering processes. However, at finite
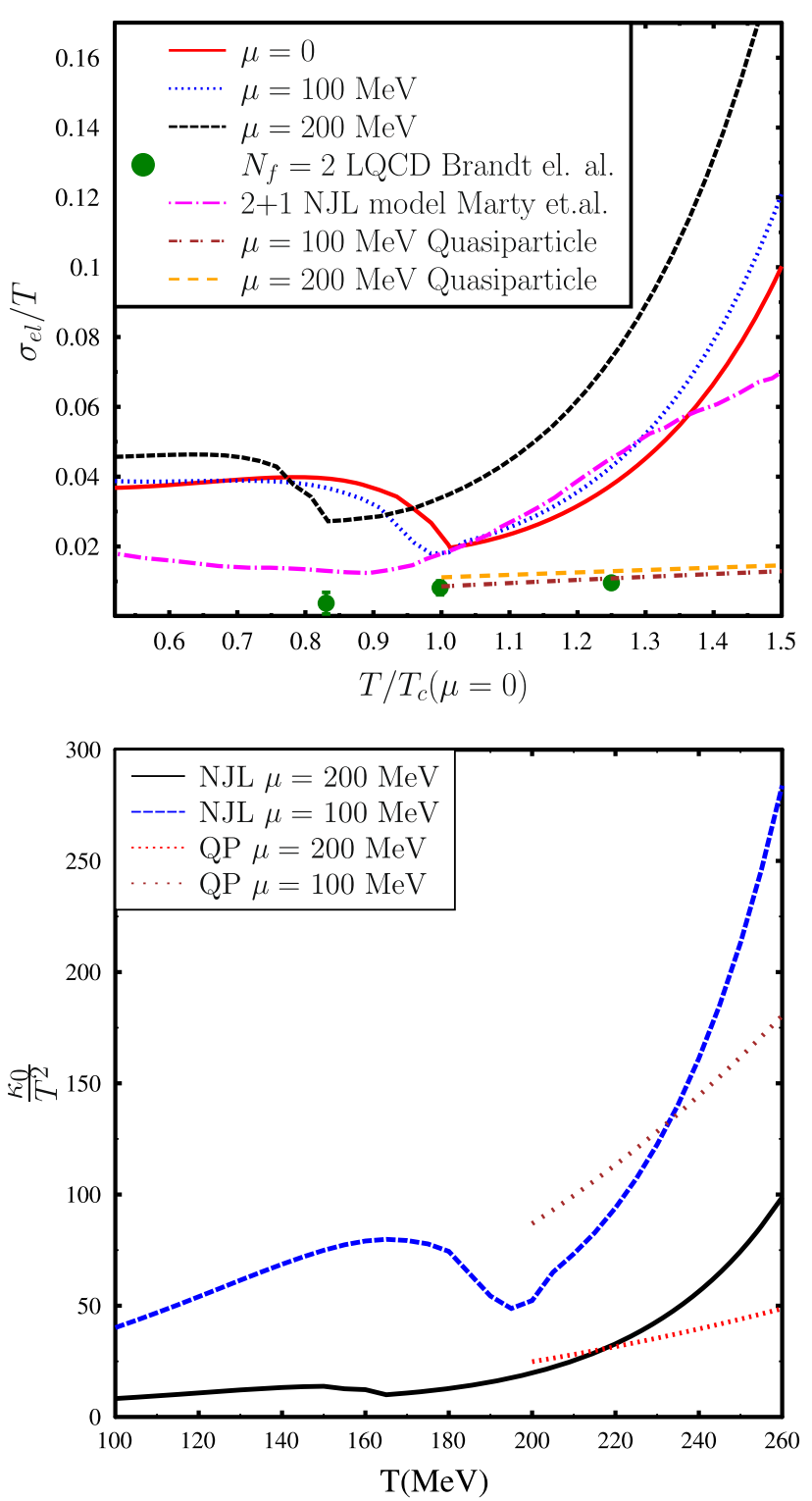

Fig. 4 Upper panel: Variation of normalized electrical conductivity $\left(\sigma_{e l} / T\right)$ with temperature for different values of the chemical potential for two flavor NJL model and the quasi particle model for the partonic matter as considered here. For comparison, we also presented the two flavor Lattice QCD (LQCD) data as given in Refs. [111,112] and 2+1 flavor NJL model results as obtained in Ref. [31]. Lower panel: Variation of normalized thermal conductivity $\left(\kappa_{0} / T^{2}\right)$ with temperature for different values of the chemical potential for NJL model and for the quasi particle model

densities, there are few antiquarks as compared to quarks so that the quarks have fewer antiquarks to scatter off. This leads to a smaller cross-section giving rise to a larger relaxation time for quarks compared to $\mu=0$ case. Due to the enhancement of quark densities at finite $\mu$, the crosssection for quark-quark scattering becomes larger resulting in a smaller relaxation time for the quarks compared to the case at vanishing chemical potential below the Mott temper- 
ature. The antiquark relaxation time, on the other hand, is always smaller compared to $\mu=0$ case as there are more quarks to scatter off at finite chemical potential.

Some discussions on the estimation of the average relaxation time is in order here. Note that one of the initial calculations in the mid-1990s as done in Ref. [113], as well as a relatively recent calculation as done in Ref. [31], where the transport coefficients for quark-gluon plasma has been estimated within the framework of QCD-inspired effective models, do not incorporate the full field theoretical methods to estimate the relaxation time. In these studies to estimate the average scattering rates or the relaxation time, one considers "integrated cross sections", by integrating the elastic cross section over the invariant energy squared with the help of a probability function (see Ref. [31] for a detailed discussion). Such an estimation of the relaxation time does not incorporate a possible nonmonotonic variation across the transition temperature/Mott temperature. On the other hand, the formalism that we have adopted here does not consider any adhoc probability function, rather we use basic definitions of scattering cross section and the thermal average of relaxation time. Also, the estimated relaxation time as obtained here and also in Ref. [81] clearly shows a nonmonotonic variation of the relaxation time across the transition temperature/Mott temperature. Such nonmonotonic variation of the relaxation time is also reflected in the expected nonmonotonic behavior of $\eta / s$ across the transition temperature [81].

Further as a validity of the Boltzmann kinetic approach within the relaxation time approximation one may look into the value of the the mean free path $\lambda_{f}=v_{f} \tau_{f}$ for a given flavor $f$, here the mean velocity $v_{f}$ can be expressed as,

$v_{f}=\frac{2 N_{c}}{(2 \pi)^{3} n_{f}} \int d^{3} p \frac{|\mathbf{p}|}{E_{p}} f\left(E_{p}\right)$.

It can be argued that at the Mott transition temperature $\lambda_{f}=1.2 \mathrm{fm}$ [81]. At the same temperature, the mass of the pion and sigma meson are of the order of $200 \mathrm{MeV}$ with the corresponding Compton wavelength $\left(\lambda_{C}\right)$ to be of the order of a Fermi. Therefore the value of the ratio $\lambda_{f} / \lambda_{C}$ is about 1.2 at the Mott transition temperature and its value increases rapidly both below and above the Mott temperature. Therefore except at the Mott transition temperature $\lambda_{f}$ can be significantly larger than $\lambda_{C}$. Thus, within the NJL model, it may not be too unreliable to use the Boltzmann equation within the relaxation time approximation except at the Mott transition temperature. Therefore we believe our analysis is not unjustified given the fact that similar approaches have been well explored by various authors also. The novelty that we are addressing is the thermoelectric properties of the QCD matter across the chiral transition scale.

In the upper panel of Fig. 4 we show the behavior of normalized electrical conductivity $\sigma_{e l} / T$ with temperature for different values of chemical potential for the present case of 2 flavor NJL model as well as the quasi particle model considered here. For comparison, we also present the results as obtained using lattice QCD for two light flavors Refs. $[111,112]$ and $2+1$ flavor NJL model results as obtained in Ref. [31]. Further, for the sake of comparison, we have taken the temperature in units of $T_{c}$ of the corresponding models. For the 2 flavor NJL model we have taken $T_{c}=T_{M}=198$ $\mathrm{MeV}$ as estimated here.

As may be observed from the figure the generic behavior of relaxation time of Fig. 3 is reflected in the behavior of electrical conductivity, having a minimum at Mott transition temperature for the two flavor NJL model shown by the solid red curve in Fig. 4. Apart from this, it is also observed that $\sigma_{e l} / T$ increases with chemical potential which we have shown by blue dotted $(\mu=100 \mathrm{MeV})$ and black dashed $(\mu=200 \mathrm{MeV})$ curves. This is because the contribution to the electrical conductivity arises dominantly from quarks rather than antiquarks at finite chemical potential, as the antiquark contribution gets suppressed due to the distribution function. This apart, there is an enhancement of the relaxation time at finite $\mu$ beyond the Mott transition. The dominant contribution to the scattering process is $u \bar{d} \rightarrow u \bar{d}$. As the $\bar{d}$ density decrease with $\mu$, this scattering process gets suppressed as compared to the case of $\mu=0$ and leads to an enhancement of relaxation time at finite chemical potential. Thus both the increase of charge carriers and an increase in relaxation time with $\mu$ lead to enhancement of electrical conductivity beyond the Mott temperature. On the other hand, below the Mott temperature, although the relaxation time decrease with chemical potential, the increase in quark number density makes the coefficient of electrical conductivity increasing with chemical potential. Further, in the hightemperature range $T>>M$, one can assume the quarks and antiquarks to be massless. In this high temperature or massless limit in the two flavor NJL model $\sigma_{e l} / T$ can be shown to be $\sigma_{e l} / T \sim T \tau \exp (\mu / T)$ (by considering only quark contribution as they are dominant at finite $\mu$ ). Therefore for a temperature range higher than the Mott transition temperature predominantly due to the increasing behavior of $\tau$ with temperature $\sigma_{e l} / T$ increases. Again at a very high temperature due to the factor of $\exp (\mu / T), \sigma_{e l} / T$ increases with chemical potential. It is clear that the order of magnitude value of the normalized electrical conductivity as obtained in the present investigation is similar to the lattice QCD (LQCD) results. However, it should be emphasized that LQCD calculations take into account the full dynamical nature of the QCD gauge fields. On the other hand, gluons are not present in the NJL model. Therefore, quantitative variation of the relaxation time and $\sigma_{e l} / T$ is not expected to be the same in NJL and LQCD calculations. Further, as compared to results of Marty et al. [31] shown by magenta dot dashed curve, the 2flavor NJL model values are of similar order near the 
transition temperature while at high temperature $\left(T / T_{c}>1.4\right)$ the two flvor NJL values are larger whereas the $2+1$ flavor values flatten out.This is because of two reasons: firstly, with $2+1$ flavors, the relaxation time decreases as there are extra channels for scatterings available that reduces the relaxation time. Further, there is a difference in the definition of relaxation time given in Ref. [31] and the present definition for the estimation of the same [81].

We have also plotted the results for the electrical conductivity estimated from the quasi particle model which remains almost constant compared to the NJL model results. The reason is, in the quasi particle models, the quasi particle masses are increasing functions of temperature and hence the thermal distribution functions get suppressed at high temperature in contrast to NJL model. Further, the magnitude of the velocity $|\mathbf{p}| / E$ also become smaller at high temperature in quasi particle model as compared to the NJL model.

Furthermore, in Ref. [114] various transport coefficients of deconfined quark matter have been studied within a different quasi particle model, namely the effective fugacity quasiparticle model. The crucial difference between the quasi particle model considered here and that in Ref. [114] lies in a different dispersion relation between the quasi particles. This is manifested in the estimation of relaxation time as well as in estimation of various transport coefficients. It should be noted that normalized electrical conductivity $\sigma_{e l} / T$ as obtained in the present investigation is quantitatively as well as qualitatively different from the same as obtained in Ref. [114]. The presence of a background scalar condensate is the fundamental difference between the NJL model and the effective fugacity quasi-particle model as discussed in Ref. [114]. Further, relaxation time plays an important role in determining the variation of any transport coefficient with temperature and chemical potential. The thermal averaged relaxation time as obtained in the effective fugacity quasi-particle model as discussed in the Ref. [114] is different (quantitatively and quantitatively) from the relaxation time obtained in the NJL model as well as the quasi particle model considered here. For a more detailed analysis of the estimation of electrical conductivity in quasi particle model as considered here and that of effective fugacity quasi particle model, we refer the interested reader to Ref. [110]. The difference stems from the difference in the single particle energy dispersion relation as compared to NJL model or the quasi particle model considered here.

In the lower panel of Fig. 4 we show the variation of the normalized thermal conductivity $\left(\kappa_{0} / T^{2}\right)$ with temperature both for NJL model and for the quasi particle model. For the NJL model, the ratio shows a nonmonotonic variation with temperature. The origin of such behavior again lies with the variation of relaxation time with temperature. Beyond the Mott temperature, the thermal conductivity increases sharply with temperature. This can be understood as fol- lows. For large temperatures, when quark masses can be neglected, it can be easily shown that the enthalpy to the net quark number density ratio goes as $\omega / n_{q} \sim T \operatorname{coth}(\mu / T)$. Also note that in the expression of the thermal conductivity $\left(E-\frac{\omega}{n_{q}}\right)^{2} \sim\left(\frac{\omega}{n_{q}}\right)^{2}$, due to the fact that single-particle energy $(E)$ is negligible as compared to the enthalpy per particle$\omega / n_{q}$. Therefore, the variation of the normalized thermal conductivity with temperature and chemical potential will be determined by the variation of relaxation time, $\omega / n_{q}$, and the distribution function. It can be shown that in the high-temperature limit or the massless limit the normalized thermal conductivity, $\kappa_{0} / T^{2}$ can be approximately expressed as, $\kappa_{0} / T^{2} \sim T \tau \exp (\mu / T)(\operatorname{coth}(\mu / T))^{2}$. Beyond the Mott transition temperature, the increasing behavior of $\tau$ essentially determines the increasing behavior of $\kappa_{0} / T^{2}$. On the other hand for $\mu<<T, \operatorname{coth}(\mu / T) \sim T / \mu$ in the leading order. Therefore in the high-temperature limit, $\kappa_{0} / T^{2}$ decreases with chemical potential. For the quasi particle model, on the otherhand, the ratio $\kappa_{0} / T^{2}$ is of the same order near the transition temperature but rises slowly with temperature compared to the NJL model which again is a reflection of increasing behaviour in the masses of the quasi particle with temperature which reduces the thermal distribution functions. Similar to $\sigma_{e l} / T$, the qualitative nature of the normalized thermal conductivity $\left(\kappa_{0} / T^{2}\right)$ as presented here is also different from the same as obtained in the Ref. [114]. This difference is again due to different nature of the dispersion relation for the single particle energies of the quasi particles in effective fugacity quasi particle model and the NJL or the quasi particle model considered here.

We next show the behavior of the Seebeck coefficient as a function of temperature for different values of quark chemical potential in the upper panel of Fig. 5 for both in NJL model and in quasi particle model. This coefficient, which is dimensionless, decreases monotonically with temperature. The variation of the Seebeck coefficient with temperature can be understood as follows. Note that this coefficient is a ratio of two quantities each of which is proportional to the relaxation time. When we consider the relaxation time as the average relaxation time, the ratio becomes independent of the average relaxation time. Note that at finite chemical potential quark contribution to the Seebeck coefficient is dominant with respect to the antiquark contribution. Therefore, contrary to the nonmonotonic variation of $\sigma_{e l} / T$ and $\kappa_{0} / T^{2}$ with temperature for NJL model, where the nonmonotonic variation has its origin stemming from the behavior of relaxation time with temperature, the variation of the Seebeck coefficient is not expected to be nonmonotonic. Further unlike other transport coefficients, the positivity of the Seebeck coefficient is not guaranteed. This is because in the expression of the Seebeck coefficient as given in Eq. (17), the integrand in the numerator has the factor which is linear in 

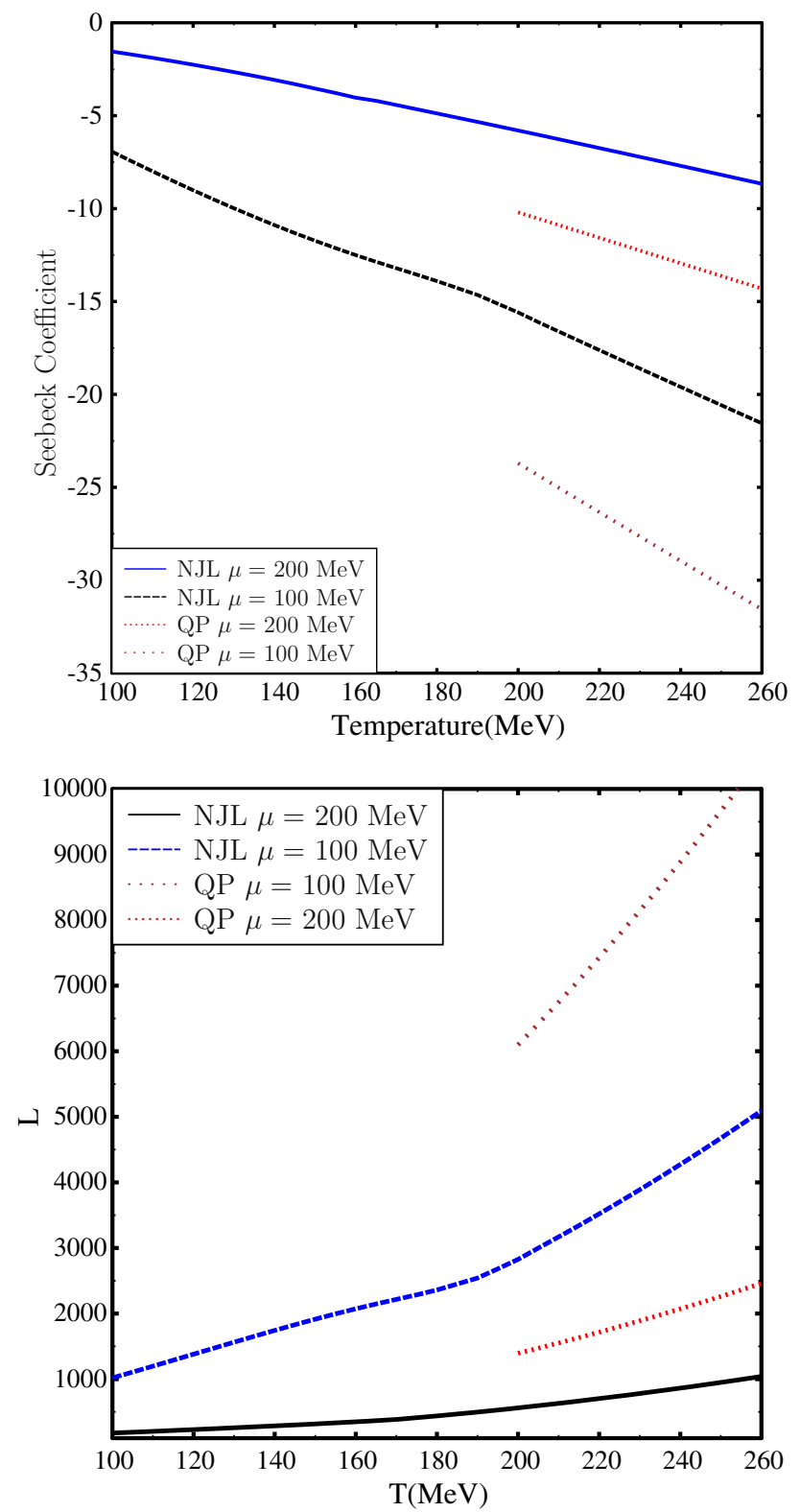

Fig. 5 Upper panel: variation of the Seebeck coefficient with temperature for different values of the chemical potential for NJL model and for quasi particle model. Lower panel: variation of the Lorenz number, $L=\kappa_{0} /\left(\sigma_{e l} T\right)$ with temperature for different values of the chemical potential in both NJL and quasi particle model

$\left(E_{a}-b_{a} \omega / n_{q}\right)$. Therefore for the quarks, this factor becomes $\left(E-\omega / n_{q}\right)$, and the single-particle energy $E$ is much smaller than $\omega / n_{q}$. Therefore the term $\left(E-\omega / n_{q}\right)$ is negative which makes the Seebeck coefficient negative. However it is important to note that the expression of thermal conductivity also contains a term $\left(E-\omega / n_{q}\right)$, but it comes as a square. Therefore positivity of the thermal conductivity is guaranteed. In the condensed matter system the Seebeck coefficient can be both positive and negative, e.g. if for electron and holes the Seebeck coefficients are opposite to each other. Further for a bipolar medium with multiple charge carriers the sign of the Seebeck coefficient depends on the range of temperature considered [102]. Similar to the case of thermal conductivity, one can do an analysis regarding the behavior of the Seebeck coefficient in the massless limit. In the massless limit, it can be shown that $S \sim-\operatorname{coth}(\mu / T)$. Therefore in the high-temperature limit, the leading order contribution to the Seebeck coefficient is $S \sim-T / \mu$. Hence with increasing temperature the Seebeck coefficient decreases, on the other hand with an increase in chemical potential Seebeck coefficient increases. In the simple analysis, we have assumed that the dominant contributions in the sum over species arise from quarks as the antiquark contributions are suppressed due to finite chemical potential in the thermal distribution function. A comment regarding $\mathrm{SU}(2)$ flavor symmetry of the NJL Lagrangian may be relevant here. The thermalisation of the medium is decided by strong interaction. Thus, the relaxation time for up and a down quarks will be same. On the otherhand, the contribution of the up quark and down quark to the Seebeck coefficient will be different as the Seebeck coefficient depend linearly on the electrical charge of the relevant species [see e.g. the numerator of the expression for Seebeck coefficient in Eq. (17)]. Thus, the contribution of the Seebeck coefficient of up quark will be twice in magnitude and opposite in sign of the down quark.

Compared to the NJL model, the behaviour of the Seebeck coefficient in the quasi particle model is qualitatively similar but quantitatively different. This can be understood from the behaviour of the electrical conductivity in the model as shown in Fig. 4. The smaller value for the electrical conductivity in quasi particle model leads to larger magnitude for the Seebeck coefficient. Further, in the quasi particle models the gluons also contribute to the enthalpy which affects the Seebeck coefficient.

In the lower panel of Fig. 5 we have plotted the ratio $L=\kappa_{0} /\left(\sigma_{e l} T\right)$, as a function of temperature for the NJL model as well as for the quasi particle model. In condensed matter systems this ratio is a constant and is known as the Lorenz number. In the present case, however, it is observed that the ratio increase monotonically with temperature. Similar to the Seebeck coefficient, in the leading order for average relaxation time the ratio $L$, is independent of relaxation time. Further, in the high temperature limit or in the massless limit $\kappa_{0} /(\sigma T) \sim(\operatorname{coth}(\mu / T))^{2}$. Therefore, in the leading order for $\mu<<T, \kappa_{0} /(\sigma T) \sim T^{2} / \mu^{2}$. Hence in the high temperature limit the ratio $L$ increases with temperature but decreases with quark chemical potential. In quasi particle model this ratio is higher compared to the NJL model as the electrical conductivity in the quasi particle description is smaller compared to that in NJL model. This ratio has also been estimated within the effective fugacity quasi particle model in Ref. [114] where, this ratio approach a constant at high temperature. This different behaviour has its origin in 
the different behaviour of the quasi particles in the effective fugacity quasi particle model as discussed earlier.

\section{Conclusion}

In the present investigation, we have estimated the Seebeck coefficient in a hot and dense partonic medium modeled by the Nambu-Jona-Lasinio model. To compare the NJL model results for the Seebeck coefficient we have also estimated the same within a quasiparticle model of the deconfined matter. We have considered thermoelectric effect arising due to temperature gradient as well as a gradient in the chemical potential. Apart from the Seebeck coefficient we have also estimated electrical conductivity, thermal conductivity, and Lorenz number associated with the Wiedemann- Franz law. Note that electrical conductivity is the response of a medium in the presence of an external electric field. $\sigma_{e l}$ also decides the time evolution of a magnetic field in a conducting medium. In the context of the effect of magnetic field on the strongly interacting medium produced in non-central heavyion collision electrical conductivity plays a crucial role. It should be emphasized that in the presence of a magnetic field simple Ohm's law gets modified and one needs to consider the anisotropic structure of the electrical conductivity tensor. All such investigations in the presence of a magnetic field should reproduce the electrical conductivity in the absence of any magnetic field, i.e. the electrical conductivity tensor should be isotropic in the absence of any magnetic field. Therefore, the estimation of electrical conductivity without any effect of the magnetic field should serve as a baseline for the studies that include the effect of magnetic field in a conducting medium.

Although electrical conductivity and thermal conductivity always remain positive, but the Seebeck coefficient is negative for the range of temperature and chemical potential considered in this investigation. Also the variation of electrical conductivity and thermal conductivity with temperature and quark chemical potential is intimately related to the variation of the relaxation time with temperature and chemical potential. But the variation of the Seebeck coefficient and the Lorenz number are not sensitive to the variation of relaxation time with temperature and quark chemical potential.

In the presence of thermoelectric effects in a conducting medium temperature gradient can be converted into an electrical current and vice versa. Seebeck coefficient physically represents the efficiency of any conducting medium to convert a temperature gradient into an electrical current. Therefore for a nonvanishing Seebeck coefficient electrical current as well as heat current gets modified. The electrical current in the presence of Seebeck effect becomes, $\mathbf{J}=\sigma_{e l} \mathcal{E}-\sigma_{e l} S \nabla T$. It is important to note that the electrical conductivity $\sigma_{e l}$ is always positive due to the constructive contributions of parti- cles and antiparticles to the electric current. Positivity of the electrical conductivity can be shown using entropy production i.e. second law of thermodynamics. By demanding that in the presence of electromagnetic field $T \partial_{\mu} s^{\mu} \geq 0$, here $s^{\mu}$ is the entropy current, it can be shown that the electrical conductivity is positive [115]. For a negative Seebeck coefficient in the presence of a positive temperature gradient the electric current enhances. Therefore the net electric current increases if the electric current due to the thermoelectric effect and the electric current due to the external electric field contributes constructively. On the other hand, the thermal conductivity in the presence of the thermoelectric effect gets modified. In the presence of a nonvanishing Seebeck coefficient, the net thermal conductivity which can be given as $\kappa=\kappa_{0}-T \sigma_{e l} S^{2}$ indicates that the nonvanishing value of the Seebeck coefficient reduces the thermal conductivity. It is important to note that the thermal conductivity is required to be positive for the theory to be consistent with the second law of thermodynamics, i.e., $T \partial_{\mu} s^{\mu} \geq 0$. Using the formalism of viscous hydrodynamics and viscous magnetohydrodynamics positivity of the electrical conductivity and the thermal conductivity has been shown explicitly [100,115]. But the contributions to the entropy current coming from the thermoelectric effects are not considered in these investigations. Therefore in the context of entropy production in the viscous hydrodynamics and magnetohydrodynamics, it will be interesting to study the effects of thermoelectric coefficients.

Thermoelectric coefficients could also be relevant in the context of the spin Hall effect (SHE). Spin Hall effect is an important ingredient for the generation of spin current and it is a key concept in spintronics. In the generation of spin current spin Hall effect plays an important role. In spin Hall effect an electric field induces a transverse spin current perpendicular to the direction of the electric field. Spin Hall effect has been investigated recently in a hot and dense nuclear matter in the context of heavy-ion collisions [116]. It has been argued that due to SHE, a spin current will be produced proportional to the electric field. This also means external electric field $\mathcal{E}$ will induce a local spin polarization and the spin polarization distribution function of fermions (antifermions) in momentum space will feature a dipole distribution. Therefore there will a spin flow in the plane transverse to the direction of the electric field. Observation of spin Hall effect may open a new direction in the exploration of the many body quantum effects in hot and dense nuclear matter. However, the life-time of the electric field originated in heavy-ion collisions could be small of the order $1 \mathrm{fm} / \mathrm{c}$. Therefore, the idea of the observation of the spin Hall effect becomes speculative. However, in the presence of nonvanishing thermoelectric coefficients any temperature gradient and/or a gradient in the chemical potential can give rise to an effective electric field which may contribute to the spin Hall effect. Therefore a detailed analysis of the thermoelec- 
tric property of the hot and dense matter produced in a heavy ion collision experiment could be relevant for spin Hall effect and needs further investigation.

Acknowledgements We thank Prof. Ajit M. Srivastava for suggesting and initiating discussions on the idea of thermoelectric coefficient in the context of heavy-ion collision. The authors would like to thank Prof. Jitesh R. Bhatt for useful discussions. The authors would also like to thank Sabyasachi Ghosh, Abhishek Atreya, Chowdhury Aminul Islam, Rajarshi Ray for many discussions on the topic of Seebeck coefficient during working group activities at WHEPP 2017, IISER Bhopal. The work of A.D. is supported by the Polish National Science Center Grant no. $2018 / 30 / \mathrm{E} / \mathrm{ST} 2 / 00432$.

Data Availability Statement This manuscript has no associated data or the data will not be deposited. [Authors' comment: The plots are based on Eq. 25, Eq. 26, Eq. 31 and Eq. 32 in the paper together with the values of the parameters given. This is sufficient to generate the plots.]

Open Access This article is licensed under a Creative Commons Attribution 4.0 International License, which permits use, sharing, adaptation, distribution and reproduction in any medium or format, as long as you give appropriate credit to the original author(s) and the source, provide a link to the Creative Commons licence, and indicate if changes were made. The images or other third party material in this article are included in the article's Creative Commons licence, unless indicated otherwise in a credit line to the material. If material is not included in the article's Creative Commons licence and your intended use is not permitted by statutory regulation or exceeds the permitted use, you will need to obtain permission directly from the copyright holder. To view a copy of this licence, visit http://creativecomm ons.org/licenses/by/4.0/.

Funded by SCOAP ${ }^{3}$.

\section{References}

1. U. Heinz, R. Snellings, Ann. Rev. Nucl. Part. Sci. 63, 123-151 (2013)

2. P. Romatschke, U. Romatschke, Phys. Rev. Lett. 99, 172301 (2007)

3. P. Kovtun, D.T. Son, A.O. Starinets, Phys. Rev. Lett. 94, 111601 (2005)

4. A. Dobado, J.M. Torres-Rincon, Phys. Rev. D 86, 074021 (2012)

5. C. Sasaki, K. Redlich, Phys. Rev. C 79, 055207 (2009)

6. C. Sasaki, K. Redlich, Nucl. Phys. A 832, 62-75 (2010)

7. F. Karsch, D. Kharzeev, K. Tuchin, Phys. Lett. B 663, 217-221 (2008)

8. S.I. Finazzo, R. Rougemont, H. Marrochio, J. Noronha, JHEP 02, 051 (2015)

9. S. Jeon, L.G. Yaffe, Phys. Rev. D 53, 5799-5809 (1996)

10. A. Bazavov et al., Phys. Rev. D 80, 014504 (2009)

11. A. Bazavov, P. Petreczky, PoS LATTICE2010:169, 2010

12. P. Bozek, Phys. Rev. C 81, 034909 (2010)

13. J.B. Rose, J.F. Paquet, G.S. Denicol, M. Luzum, B. Schenke, S. Jeon, C. Gale, Nucl. Phys. A 931, 926-930 (2014)

14. K. Tuchin, Phys. Rev. C 83, 017901 (2011)

15. K. Tuchin, Phys. Rev. C 82, 034904 (2010)

16. G. Inghirami, L.D. Zanna, A. Beraudo, M.H. Moghaddam, F. Becattini, M. Bleicher, Eur. Phys. J. C 76, 659 (2016)

17. A. Das, S.S. Dave, P.S. Saumia, A.M. Srivastava, Phys. Rev. C 96, 034902 (2017)
18. M. Greif, C. Greiner, G.S. Denicol, Phys. Rev. D 93, 096012 (2016)

19. M. Greif, I. Bouras, C. Greiner, Z. Xu, Phys. Rev. D 90, 094014 (2014)

20. A. Puglisi, S. Plumari, V. Greco, Phys. Lett. B 751, 326-330 (2015)

21. A. Puglisi, S. Plumari, V. Greco, Phys. Rev. D 90, 114009 (2014)

22. W. Cassing, O. Linnyk, T. Steinert, V. Ozvenchuk, Phys. Rev. Lett. 110, 182301 (2013)

23. T. Steinert, W. Cassing, Phys. Rev. C 89, 035203 (2014)

24. G. Aarts, C. Allton, A. Amato, P. Giudice, S. Hands, J.I. Skullerud, JHEP 02, 186 (2015)

25. G. Aarts, C. Allton, J. Foley, S. Hands, S. Kim, Phys. Rev. Lett. 99, 022002 (2007)

26. A. Amato, G. Aarts, C. Allton, P. Giudice, S. Hands, J.I. Skullerud, Phys. Rev. Lett. 111, 172001 (2013)

27. S. Gupta, Phys. Lett. B 597, 57-62 (2004)

28. H.T. Ding, A. Francis, O. Kaczmarek, F. Karsch, E. Laermann, W. Soeldner, Phys. Rev. D 83, 034504 (2011)

29. O. Kaczmarek, M. Müller, PoS LATTICE2013:175 (2014)

30. S. Qin, Phys. Lett. B 742, 358-362 (2015)

31. R. Marty, E. Bratkovskaya, W. Cassing, J. Aichelin, H. Berrehrah, Phys. Rev. C 88, 045204 (2013)

32. D.F. Fraile, A.G. Nicola, Phys. Rev. D 73, 045025 (2006)

33. D.E. Kharzeev, L.D. McLerran, H.J. Warringa, Nucl. Phys. A 803, 227-253 (2008)

34. V. Skokov, AYu. Illarionov, V. Toneev, Int. J. Mod. Phys. A 24, 5925-5932 (2009)

35. H. Li, X. Sheng, Q. Wang, Phys. Rev. C 94, 044903 (2016)

36. G. Inghirami, M. Mace, Y. Hirono, L.D. Zanna, D.E. Kharzeev, M. Bleicher, Eur. Phys. J. C 80, 293 (2020)

37. G. Inghirami, L.D. Zanna, A. Beraudo, M.H. Moghaddam, F. Becattini, M. Bleicher, J. Phys. Conf. Ser. 1024, 012043 (2018)

38. M. Shokri, N. Sadooghi, Phys. Rev. D 96, 116008 (2017)

39. M. Shokri, N. Sadooghi, JHEP 11, 181 (2018)

40. S.M.A. Tabatabaee, N. Sadooghi, Phys. Rev. D 101, 076022 (2020)

41. D.E. Kharzeev, K. Landsteiner, A. Schmitt, H.U. Yee, Strongly interacting matter in magnetic fields': an overview. Lect. Notes Phys. 871, 1 (2013)

42. M. Greif, J.A. Fotakis, G.S. Denicol, C. Greiner, Phys. Rev. Lett. 120, 242301 (2018)

43. M. Prakash, M. Prakash, R. Venugopalan, G. Welke, Phys. Rep. 227, 321-366 (1993)

44. A. Wiranata, M. Prakash, Phys. Rev. C 85, 054908 (2012)

45. P. Chakraborty, J.I. Kapusta, Phys. Rev. C 83, 014906 (2011)

46. A.S. Khvorostukhin, V.D. Toneev, D.N. Voskresensky, Nucl. Phys. A 845, 106-146 (2010)

47. S. Plumari, A. Puglisi, F. Scardina, V. Greco, Phys. Rev. C 86, 054902 (2012)

48. M.I. Gorenstein, M. Hauer, O.N. Moroz, Phys. Rev. C 77, 024911 (2008)

49. J. Noronha-Hostler, J. Noronha, C. Greiner, Phys. Rev. C 86, 024913 (2012)

50. S.K. Tiwari, P.K. Srivastava, C.P. Singh, Phys. Rev. C 85, 014908 (2012)

51. S. Ghosh, A. Lahiri, S. Majumder, R. Ray, S.K. Ghosh, Phys. Rev. C 88, 068201 (2013)

52. R. Lang, N. Kaiser, W. Weise, Eur. Phys. J. A 51, 127 (2015)

53. S. Ghosh, G. Krein, S. Sarkar, Phys. Rev. C 89, 045201 (2014)

54. A. Wiranata, V. Koch, M. Prakash, X.N. Wang, J. Phys. Conf. Ser. 509, 012049 (2014)

55. A. Wiranata, M. Prakash, P. Chakraborty, Cent. Eur. J. Phys. 10, 1349-1351 (2012)

56. J. Noronha-Hostler, J. Noronha, C. Greiner, Phys. Rev. Lett. 103, 172302 (2009) 
57. G.P. Kadam, H. Mishra, Nucl. Phys. A 934, 133-147 (2014)

58. G.P. Kadam, Mod. Phys. Lett. A 30, 1550031 (2015)

59. S. Ghosh, Int. J. Mod. Phys. A 29, 1450054 (2014)

60. J.B. Rose, J.M. Torres-Rincon, A. Schäfer, D.R. Oliinychenko, H. Petersen, Phys. Rev. C 97, 055204 (2018)

61. C. Wesp, A. El, F. Reining, Z. Xu, I. Bouras, C. Greiner, Phys. Rev. C 84, 054911 (2011)

62. G.S. Denicol, H. Niemi, I. Bouras, E. Molnar, Z. Xu, D.H. Rischke, C. Greiner, Phys. Rev. D 89, 074005 (2014)

63. J.I. Kapusta, J.M. Torres-Rincon, Phys. Rev. C 86, 054911 (2012)

64. P. Ao, arXiv:cond-mat/9505002

65. M. Matusiak, K. Rogacki, T. Wolf, Phys. Rev. B 97, 220501 (2018)

66. C.S. Yadav, M.K. Hooda, arXiv:1704.07194

67. O. Cyr-Choinière, S. Badoux, G. Grissonnanche, B. Michon, S.A.A. Afshar, S. Fortier, D. LeBoeuf, D. Graf, J. Day, D.A. Bonn, W.N. Hardy, R. Liang, N. Doiron-Leyraud, L. Taillefer, Phys. Rev. X 7, 031042 (2017)

68. S. Sergei, JETP Lett. 67, 680-684 (1998)

69. M.M. Wysokinski, J. Spalek, J. Appl. Phys. 113, 163905 (2013)

70. K.P. Wójcik, I. Weymann, Phys. Rev. B 89, 165303 (2014)

71. K. Seo, S. Tewari, Phys. Rev. B 90, 174503 (2014)

72. P. Dutta, A. Saha, A.M. Jayannavar, Phys. Rev. B 96, 115404 (2017)

73. A. Das, H. Mishra, R.K. Mohapatra, Phys. Rev. D 102, 014030 (2020)

74. J.R. Bhatt, A. Das, H. Mishra, Phys. Rev. D 99, 014015 (2019)

75. D. Dey, B.K. Patra, arXiv:2004.03149

76. H.-X. Zhang, arXiv:2004.08767

77. P. Singha, A. Abhishek, G. Kadam, S. Ghosh, H. Mishra, J. Phys. G 46, 015201 (2019)

78. A. Abhishek, H. Mishra, S. Ghosh, Phys. Rev. D 97, 014005 (2018)

79. B. Singh, A. Abhishek, S.K. Das, H. Mishra, Phys. Rev. D 100, 114019 (2019)

80. C. Ratti, M.A. Thaler, W. Weise, Phys. Rev. D 73, 014019 (2006)

81. P. Deb, G.P. Kadam, H. Mishra, Phys. Rev. D 94, 094002 (2016)

82. P. Rehberg, S.P. Klevansky, J. Hufner, Phys. Rev. C 53, 410-429 (1996)

83. Y. Tsue, J. da Providencia, C. Providencia, M. Yamamura, Prog. Theor. Phys. 128, 507-522 (2012)

84. T. Maruyama, E. Nakano, T. Tatsumi, Horizons in World Physics, Chapter 7, vol. 276 (Nova Science, New York, 2011)

85. D.P. Menezes, M.B. Pinto, S.S. Avancini, A.P. Martinez, C. Providencia, Phys. Rev. C 79, 035807 (2009)

86. B. Chatterjee, H. Mishra, A. Mishra, Phys. Rev. D 84, 014016 (2011)

87. T. Mandal, P. Jaikumar, S. Digal, arXiv:0912.1413
88. T. Mandal, P. Jaikumar, Phys. Rev. C 87045208 (2013)

89. T. Mandal, P. Jaikumar, Phys. Rev. D 94, 074016 (2016)

90. M. Coppola, P. Allen, A.G. Grunfeld, N.N. Scoccola, Phys. Rev. D 96, 056013 (2017)

91. M. Bluhm, QCD equation of state of hot deconfined matter at finite baryon densities: a quasi particle perspective, $\mathrm{Ph} . \mathrm{D}$. thesis, Technical University of Dresden 2008

92. A. Peshier, B. kampfer, Phys. Rev C 61, 045203 (2000)

93. A. Peshier, B. Kampfer, G. Soff, Phys. Rev. D 66, 094003 (2002)

94. R.A. Schneider, W. Weise, Phys. Rev. C 64, 055201 (2001)

95. S. Koothottil, V.M. Bannur, Phys. Rev. C 102, 015206 (2020)

96. P.K. Srivastava, S.K. Tiwari, C.P. Singh, Phys. Rev. D 82, 014023 (2010)

97. M.V. Bannur, JHEP 09, 046 (2007)

98. A.S. Khvorostukhin, V.D. Toneev, D.N. Voskresensky, Nucl. Phys. A 915, 158-169 (2013)

99. P. Zhuang, J. Hufner, S.P. Klevansky, L. Neise, Phys. Rev. D 51, 3728-3738 (1995)

100. S. Gavin, Nucl. Phys. A 435, 826-843 (1985)

101. G.P. Kadam, H. Mishra, L. Thakur, Phys. Rev. D 98, 114001 (2018)

102. L.Y. Zhou, Q. Zheng, L.H. Bao, W.J. Liang, Chin. Phys. Lett. 37, $017301(2020)$

103. M. Buballa, Phys. Rep. 407, 205-376 (2005)

104. L. Thakur, P.K. Srivastava, G.P. Kadam, M. George, H. Mishra, Phys. Rev. D 95, 096009 (2017)

105. M.V. Bannur, Phys. Rev. C 75, 044905 (2007)

106. L.L. Zhu, C.B. Yang, Nucl. Phys. A 831, 49-58 (2009)

107. M.I. Gorenstein, S.N. Yang, Phys. Rev. D 52, 5206 (1995)

108. A. Hosoya, K. Kajantie, Nucl. Phys. B 250, 666 (1985)

109. H. Berrehrah, E. Bratkovskaya, W. Cassing, P.B. Gossiaux, J. Aichelin, M. Bleicher, Phys. Rev. C 89, 054901 (2014)

110. A. Das, H. Mishra, R.K. Mohapatra, Phys. Rev. D 101, 034027 (2020)

111. B.B. Brandt, A. Francis, B. Jaeger, H.B. Meyer, Phys. Rev. D 93, 054510 (2016)

112. G. Aarts, A. Nikolaev, Eur. Phys. J. A 57, 118 (2021)

113. P. Rehberg, S.P. Klevansky, J. Hufner, Nucl. Phys. A 608, 356-388 (1996)

114. S. Mitra, V. Chandra, Phys. Rev. D 96, 094003 (2017)

115. X.G. Huang, M. Huang, D.H. Rischke, A. Sedrakian, Phys. Rev. D 81, $045015(2010)$

116. S.Y.F. Liu, Y. Yin, Phys. Rev. D 104, 054043 (2021) 